\title{
MicroRNA-891a-5p is a novel biomarker for non-small cell lung cancer and targets HOXA5 to regulate tumor cell biological function
}

\author{
NIANQING WAN and JIANXIAO ZHENG \\ Department of Integrated Traditional Chinese and Western Medicine, \\ Yueqing People's Hospital, Yueqing, Zhejiang 325600, P.R. China
}

Received September 2, 2020; Accepted February 23, 2021

DOI: 10.3892/ol.2021.12768

\begin{abstract}
Numerous studies have shown that the dysregulation of microRNA (miRNA/miR) is an important factor in the pathogenesis of lung cancer. However, the role of miR-891a-5p in non-small cell lung cancer (NSCLC) remains unclear. Therefore, the present study aimed to examine the clinical value and biological function of miR-891a-5p in NSCLC. The mRNA expression level of miR-891a-5p in NSCLC was determined using reverse transcription-quantitative PCR and was used to determine the diagnostic value of miR-891a-5p, by creating a receiver operating characteristic curve. Kaplan-Meier and Cox regression analyses were used to evaluate its prognostic value in patients with NSCLC. Furthermore, cell experiments were performed to investigate the underlying mechanisms and functional role of miR-891a-5p in NSCLC progression. The results indicated that miR-891a-5p expression level was significantly higher in serum and tissues from patients with NSCLC and NSCLC cell lines. In addition, serum miR-891a-5p was found to have a diagnostic value in patients with NSCLC, and the increase in the expression level of miR-891a-5p in tumor tissues was associated with differentiation, and the tumor, node and metastases stages of cancer, which could be used for NSCLC prognosis. In addition, the experiments revealed that NSCLC cell proliferation, invasion and migration were significantly increased by the overexpression of miR-891a-5p and were significantly reduced by its downregulation. Furthermore, a luciferase reporter assay and the protein expression levels of HOXA5 showed that HOXA5 might be a miR-891a-5p target gene. In summary, the results indicated that high miR-891a-5p expression level could be a novel biomarker in patients with NSCLC and that it promoted tumor cell proliferation, invasion
\end{abstract}

Correspondence to: Dr Nianqing Wan, Department of Integrated Traditional Chinese and Western Medicine, Yueqing People's Hospital, 338 Qingyuan Road, Chengnan Street, Yueqing, Zhejiang 325600, P.R. China

E-mail: medicine2043@163.com

Key words: miR-891a-5p, HOXA5, non-small cell lung cancer, biological function, clinical significance and migration. HOXA5 may be a target of miR-891a-5p, which may mediate miR-891a-5p function in NSCLC.

\section{Introduction}

Lung cancer is the most common malignancy and the leading cause of cancer-associated mortality, worldwide (1). More than ninety million people worldwide are at risk of developing lung cancer, which has been recognized as a major health problem for numerous years (2). According to the cancer statistics by the National Central Cancer Registry of China in China in 2015, non-small lung cancer (NSCLC) accounts for $\sim 80 \%$ of all lung cancer (3). More than $75 \%$ of lung cancer cases are advanced at diagnosis, as there is no practical way to screen a large number of people at risk (4). Despite recent advances in the understanding of the molecular biology of the lungs and the treatment of lung cancer, the prognosis of this malignancy remains poor (5). Currently, the 5-year overall survival rate of lung cancer remains unsatisfactory (6). Based on these TNM groupings, current 5-year survival estimates in NSLCC ranged from $73 \%$ in stage IA disease to $13 \%$ in stage IV disease in 2016 (7). Therefore, accurate diagnosis, improved prognosis, and effective treatment are urgently required to improve the treatment of NSCLC.

MicroRNAs (miRNAs/miR) are a class of short non-coding ribonucleic acid molecules, $\sim 23$ nucleotides in length, that play important regulatory roles in gene expression at the post-transcriptional level (8). Accumulating evidence indicates that altered expression levels of miRNA is crucial for cancer initiation and progression and functions as tumor suppressive or oncogenic miRNA $(9,10)$. miRNAs play important roles in regulating cell proliferation, migration, invasion, differentiation and apoptosis (11). In addition, miRNAs have been found to be a potential non-invasive biomarker (12). Recently, miR-891a-5p was found to be increased in lung adenocarcinoma cells compared with that in normal lung cells, and miR-891a-5p could enhance tumor cell proliferation (13). In addition, a study by Lee et al (14) also reported that the mRNA expression level of miR-891a-5p was significantly higher in patients with NSCLC. However, the clinical value of miR-891a-5p in NSCLC remains unclear and its regulatory role on tumor cell invasion and migration. 
In the present study, the expression level of miR-891a-5p in serum and tissue samples from patients with NCSLC and in NSCLC cell lines was investigated, and the diagnostic and prognostic value of miR-891a-5p was also determined. Furthermore, the effects of miR-891a-5p on NSCLC cell proliferation, migration and invasion were investigated using in vitro experiments, and the potential mechanisms of miR-891a-5p in the development of NSCLC was initially determined by analyzing the potential target genes.

\section{Materials and methods}

Patients and clinical sample collection. A total of 120 patients with NSCLC were included in the present study, who were pathologically diagnosed with NSCLC and underwent resection surgery between April 2011 and March 2014 at Yueqing People's Hospital (Yueqing, China). None of the patients received any anti-tumor therapy and the electronic medical record data of all the patients was complete. In addition, 68 ageand sex-matched healthy volunteers were recruited during the same time period as controls. The controls had routine physical examinations at Yueqing People's Hospital (Yueqing, China) and no history of malignancy. Before the patients underwent surgery, venous blood samples were collected and were immediately centrifuged for serum extraction. Blood samples were collected from all participants and immediately centrifuged at $1,500 \mathrm{x} \mathrm{g}$ for $10 \mathrm{~min}$ at $4^{\circ} \mathrm{C}$ for serum extraction. A total of 120 paired NSCLC and adjacent normal tissues, which were at least $3 \mathrm{~cm}$ away from the edge of tumors, were collected at the time of surgery and were frozen using liquid nitrogen and stored at $-80^{\circ} \mathrm{C}$. Demographic and clinicopathological characteristics, as well as survival information, from a 5-year follow-up survey (range, 0-60 months), were recorded for the subsequent analyses. Cases who died of other events, such as diseases other than NSCLC or an accident were excluded from the study. The experimental procedures were approved by the Ethics Committee of Yueqing People's Hospital (Yueqing, China; approval no. 0112983).

Cell culture and transfection. The NSCLC cell lines (H1299, HCC827, H460 and A549) and the normal lung cell line (NHBE) were purchased from The Cell Bank of Chinese Academy of Sciences. All the cells were incubated in Dulbecco's modified Eagle's medium, supplemented with $10 \%$ fetal bovine serum (FBS) (all from Invitrogen; Thermo Fisher Scientific, Inc.) at $37^{\circ} \mathrm{C}$ in a humidified atmosphere with $5 \% \mathrm{CO}_{2}$.

Cell transfection was used to increase or decrease the expression level of miR-891a-5p, using miR-891a-5p mimics and miR-891a-5p inhibitor. These and their respective negative controls (mimic NC and inhibitor NC, respectively) were synthesized by Shanghai GenePharma Co., Ltd. and transfected into the NSCLC cells using Lipofectamine ${ }^{\circledR} 2000$ (Invitrogen; Thermo Fisher Scientific, Inc.). The miR concentration and sequences were as follows: $50 \mathrm{nM}$ miR-891a-5p mimic, 5'-UGC AACGAACCUGAGCCACUGA-3'; 100 nM miR-891a-5p inhibitor, 5'-UCAGUGGCUCAGGUUCGUUGCA-3'; $50 \mathrm{nM}$ mimic NC, 5'-UUCUCCGAACGUGUCACGU-3'; and $100 \mathrm{nM}$ inhibitor NC 5'-CAGUACUUUUGUGUAGUACAA-3'. The cells transfected with the transfection reagents only, were used as the control group. Cell transfection was performed for $6 \mathrm{~h}$ at $37^{\circ} \mathrm{C}$, then culture medium was replaced and the cells were used for subsequent analyses $48 \mathrm{~h}$ after transfection.

RNA extraction and reverse transcription-quantitative $P C R$ (RT-qPCR). Total RNA was extracted from the fresh tissue, serum samples and cells using TRIzol ${ }^{\circledR}$ (Invitrogen; Thermo Fisher Scientific, Inc.), then the RNA was reverse transcribed into single-stranded cDNA using the PrimeScript reverse transcriptase kit (Takara Bio, Inc.) according to the manufacturer's guidelines. The mRNA expression level of miR-891a-5p and HOXA5 was detected using qPCR, and a SYBR-Green I Master Mix kit (Invitrogen; Thermo Fisher Scientific, Inc.) on a 7500 Real-Time PCR System (Applied Biosystems; Thermo Fisher Scientific, Inc.). U6 was used as an endogenous control for miR-891a-5p and GAPDH was used as an endogenous control for HOXA5. The thermocycling conditions were as follows: initial denaturation at $95^{\circ} \mathrm{C}$ for $10 \mathrm{~min}$, then 40 cycles of denaturation at $95^{\circ} \mathrm{C}$ for $30 \mathrm{sec}$, annealing at $60^{\circ} \mathrm{C}$ for $20 \mathrm{sec}$ and extension at $72^{\circ} \mathrm{C}$ for $15 \mathrm{sec}$. The oligonucleotide primer sequences were as follows: miR-891a-5p forward, 5'-GCCGAG TCAGUGGCTCAGGT-3' and reverse, 5'-CTCAACTGGTGT CGTGGA-3'; HOXA5 forward, 5'-AGCCACAAATCAAGGAC ACA-3' and reverse, 5'-GCTCGCTCACGGAACTATG-3'; U6 forward, 5'-GCTTCGGCAGCACATATACTAAAAT-3' and reverse: 5'-CGCTTCACGAATTTGCGTGTCAT-3'; and GAPDH, forward: 5'- TGCACCACCAACTGCTTAGC-3' and reverse, 5'-GGCATGCACTGTGGTCATGAG-3'. The final expression value was calculated using the ${ }^{2-\Delta \Delta \mathrm{Cq}}$ method (15).

Cell proliferation analysis. A Cell Counting Kit-8 (CCK-8) assay was used to detect the proliferation of the cells. The cells were seeded into 96-well plate $\left(2 \times 10^{3}\right.$ cells/well) and incubated for $0,24,48$ and $72 \mathrm{~h}$. Then, $10 \mu \mathrm{lCCK}-8$ reagent (Beyotime Institute of Biotechnology) was added to each well and further incubated for $2 \mathrm{~h}$. The optical density was measured at $450 \mathrm{~nm}$ using a microplate reader.

Transwell assay. Transwell chambers (Corning Inc.) were used to the measure the migration and invasion of the NSCLC cells. The Transwell chambers precoated with Matrigel (Corning Inc.) at $37^{\circ} \mathrm{C}$ for $1 \mathrm{~h}$ were used for the invasion assays, while the chambers without Matrigel coating were used for the migration assays. The transfected cells, at a density of $2 \times 10^{5}$ cells/chamber, were seeded into the upper chambers with serum-free medium, while culture medium, supplemented with $10 \%$ FBS, was added to the lower chambers, as a chemoattractant. After incubation at $37^{\circ} \mathrm{C}$ for $48 \mathrm{~h}$, the cells in the lower chambers were stained using $0.1 \%$ crystal violet at room temperature for $20 \mathrm{~min}$ and counted under an inverted light microscope (Olympus Corporation; magnification, x200).

Dual-luciferase reporter assay. Based on bioinformatics analysis, potential target genes can be predicted using the TargetScan online tool (http://www.targetscan.org/vert_72)(16). The wild-type (WT) and mutant type (MUT) of the HOXA5 3'-untranslated region (UTR) sequences, that contained the binding site of miR-891a-5p, were incorporated into the luciferase reporter vector, pGL3-luciferase (Promega Corporation). The recombinant vectors were co-transfected into the tumor cells with miR-891a-5p mimics, miR-891a-5p inhibitor or their 

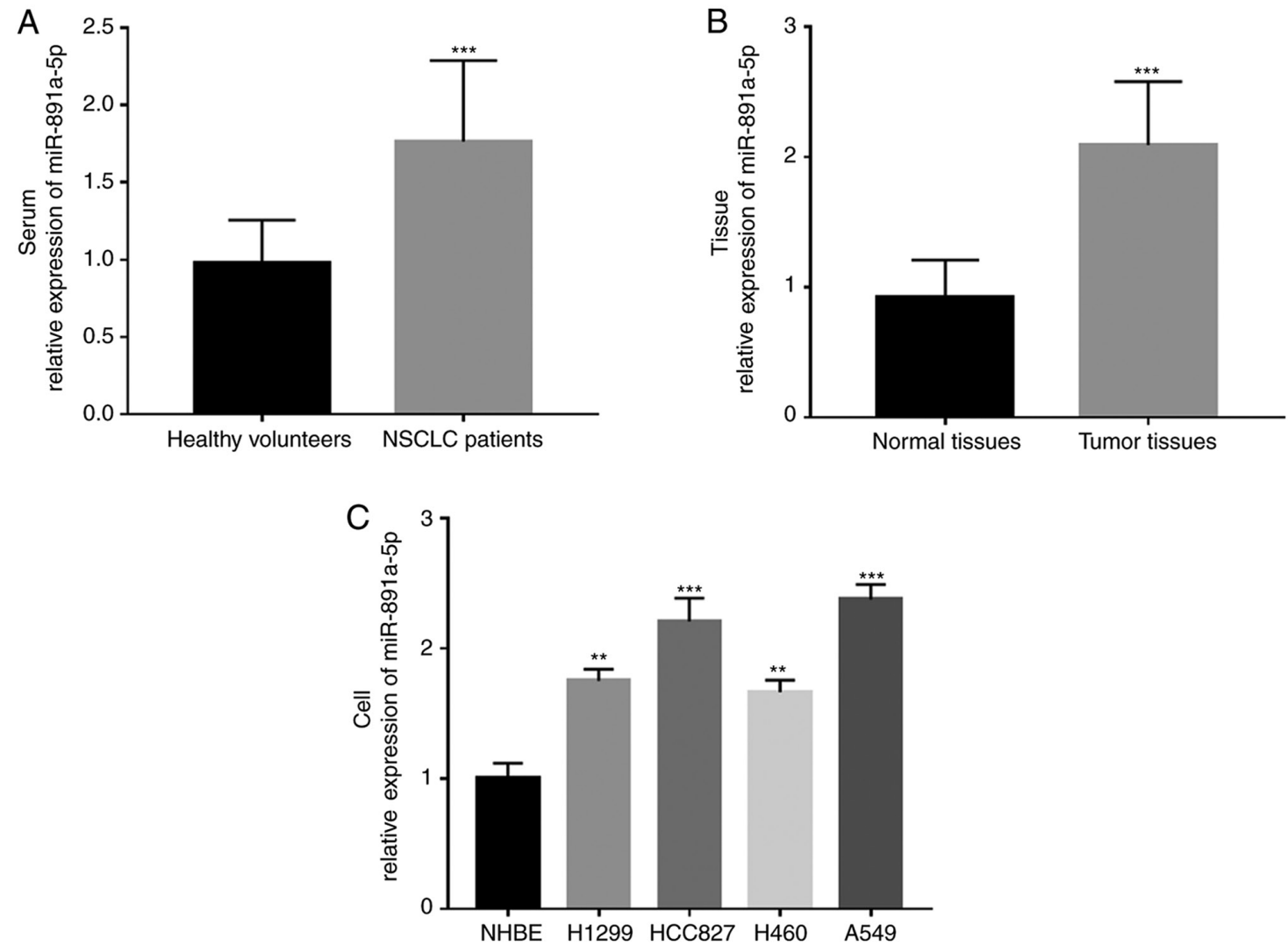

Figure 1. Expression level of miR-891a-5p in NSCLC clinical samples and cell lines. Expression level of miR-891a-5p in (A) serum and (B) tumor tissues was increased in patients NSCLC compared with that in the healthy controls and normal tissues, respectively. (A) ${ }^{* * *} \mathrm{P}<0.001 \mathrm{vs}$. healthy controls; (B) ${ }^{* * *} \mathrm{P}<0.001$ vs. normal tissues. (C) Increased expression level of miR-891a-5p in four NSCLC cell lines compared with that in the normal lung cell line. ${ }^{* *} \mathrm{P}<0.01$ and ${ }^{* * *} \mathrm{P}<0.001$ vs. NHBE. miR, microRNA; NSCLC, non-small cell lung cancer.

respective NCs, which sequences are the same as the ones used for transfection, using Lipofectamine ${ }^{\circledR} 2000$. The miR concentration and sequences were as follows: $50 \mathrm{nM}$ miR-891a-5p mimic, 5'-UGCAACGAACCUGAGCCACUGA-3'; $100 \mathrm{nM}$ miR-891a-5p inhibitor, 5'-UCAGUGGCUCAGGUUCGUU GCA-3'; 50 nM mimic NC, 5'-UUCUCCGAACGUGUCA CGU-3'; and 100 nM inhibitor NC 5'-CAGUACUUUUGUGUA GUACAA-3'. After incubation for $48 \mathrm{~h}$, the relative luciferase activity was analyzed using a Dual-Luciferase Reporter Assay System (Promega Corporation) and normalized to Renilla luciferase activity.

Statistical analysis. Statistical analyses were either performed using SPSS v21.0 (IBM Corp.) or GraphPad v7.0 (GraphPad Software, Inc.). Data are presented as the mean \pm SD. The differences in the miR-891a-5p expression level in serum samples between patients with NSCLC and the healthy controls were analyzed using an unpaired t-test, while the differences in the expression level of miR-891a-5p and HOXA5 between tumor and adjacent normal tissues were analyzed using a paired t-test. The differences between multiple groups were compared using one-way analysis of variance and a Tukey's post hoc test. The patients were divided into the miR-891a-5p high expression group $(n=62)$ and miR-891a-5p low expression group $(n=58)$ according to the mean value of miR-891a-5p expression levels in NSCLC tissues (2.091). A $\chi^{2}$ test was used to evaluate the association between miR-891a-5p expression level and the clinicopathological characteristics of the patients with NSCLC. According to the serum expression levels of miR-891a-5p, receiver operating characteristic (ROC) curve was plotted to evaluate its diagnostic value, the optimal cut-off value was calculated according to the Youden index, and the corresponding cut-off value when the Youden index was maximal was the optimal diagnostic cut-off value (Youden index $=$ Sensitivity + Specificity- 1). The Kaplan-Meier survival method and multivariate Cox regression analyses were used to examine the prognostic value of miR-891a-5p. Pearson's correlation analysis was used to analyze the correlation between the expression of miR-891a-5p and HOXA5. $\mathrm{P}<0.05$ was considered to indicate a statistically significant difference.

\section{Results}

Expression level of miR-891a-5p in NSCLC. To further understand the role of miR-891a-5p in NSCLC, the mRNA expression levels of miR-891a-5p in the NSCLC serum and tissue samples, and the NSCLC cells lines was determined using RT-qPCR. It was found that the mRNA expression levels of miR-891a-5p were significantly increased in the serum and tissue samples from the patients with NSCLC compared with that in the healthy or adjacent normal controls, respectively (both $\mathrm{P}<0.001$ ) (Fig. 1A and B). Meanwhile, the mRNA expression levels of miR-891a-5p in the NSCLC cell lines were also investigated and it was found that the expression level of miR-891a-5p was significantly higher in the NSCLC cell lines 

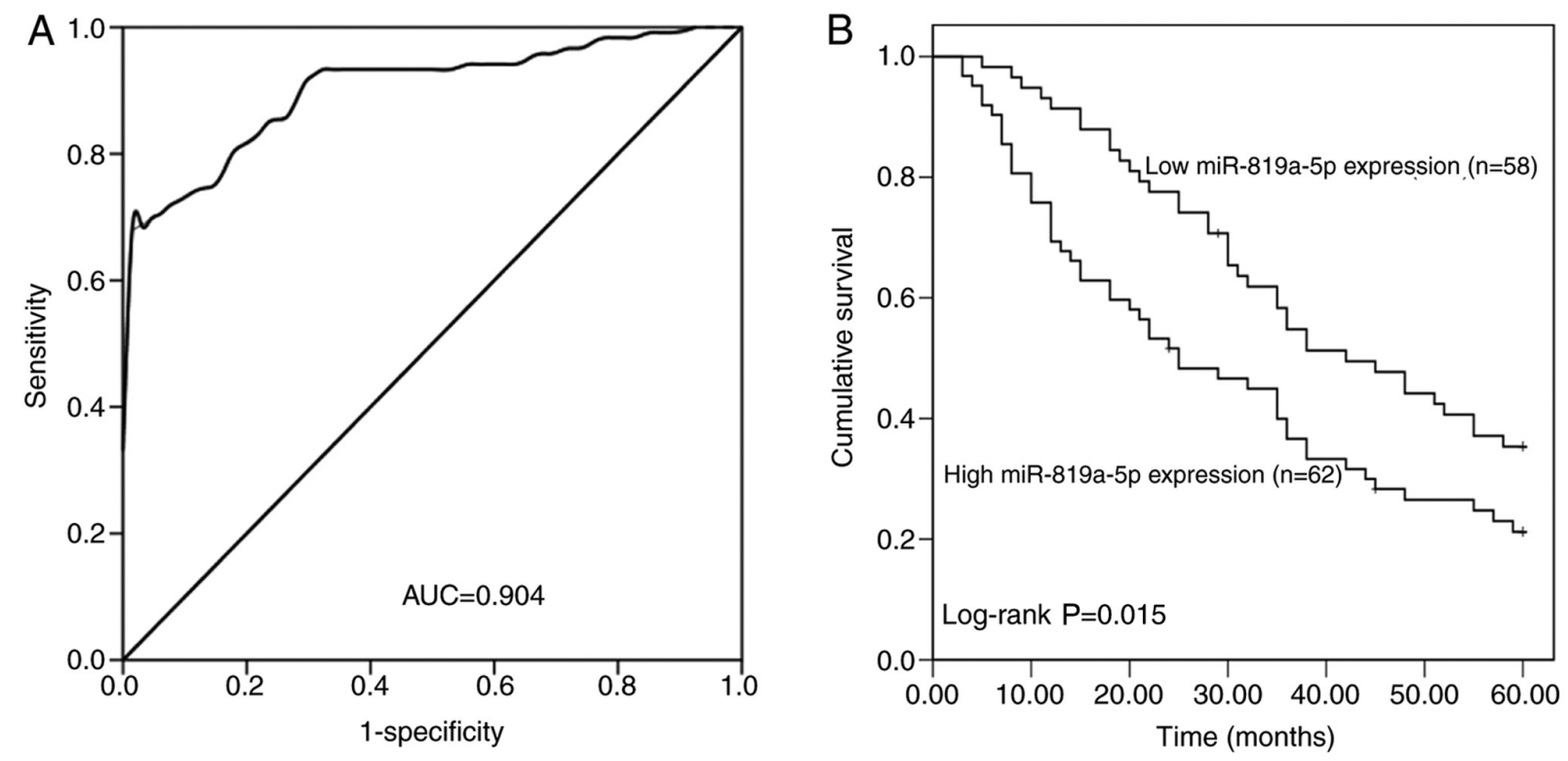

Figure 2. Clinical significance of miR-891a-5p in the diagnosis and prognosis of NSCLC. (A) A receiver operating characteristic curve was constructed using the serum miR-891a-5p expression level. (B) Kaplan-Meier survival curve. Compared with that in patients with low expression of miR-891a-5p, patients with high expression levels of miR-891a-5p had a shorter survival time. AUC, area under the curve; miR, microRNA; NSCLC, non-small cell lung cancer.

(H1299, HCC827, H460 and A549) compared with that in the normal NHBE cells (all $\mathrm{P}<0.01$ ) (Fig. 1C).

Association between the expression level of miR-891a-5p and the clinicopathological features of patients with NSCLC. Data analysis was performed to determine the association between the expression level of miR-891a-5p in the NSCLC tissues and the clinicopathological data of the patients, and to investigate the role of miR-891a-5p in the development of NSCLC. Firstly, the mean expression value of miR-891a-5p (2.091) in NSCLC tissues was used as the cutoff value to classify the patients into low and high miR-891a-5p expression groups. It was found that the miR-891a-5p expression level was associated with lymph node metastasis $(\mathrm{P}=0.029)$, differentiation $(\mathrm{P}=0.041)$ and TNM stage $(\mathrm{P}=0.019$; Table $\mathrm{I})$. In contrast, there was no association between the expression level of miR-891a-5p and the other parameters, such as tumor size, sex, age and smoking history (all $\mathrm{P}>0.05)$.

Clinical significance of miR-891a-5p in the diagnosis and prognosis of NSCLC. The expression levels of miR-891a-5p were increased in the NSCLC tissue and serum samples, and its diagnostic and prognostic significance in patients with NSCLC was further investigated. By analyzing the mRNA expression level of miR-891a-5p in the serum, a ROC curve was constructed (Fig. 2A), and the area under the cure (AUC) was found to be 0.904 , which demonstrated that miR-891a-5p had high diagnostic value. With a cut-off value of 1.236 , the sensitivity was $82.5 \%$ and the specificity was $80.9 \%$, which was the optimal relative expression value to distinguish the patients with NSCLC from the healthy controls.

Kaplan-Meier survival curves (Fig. 2B) were plotted to evaluate the association between the mRNA expression level of miR-891a-5p and the overall survival rate of the patients with NSCLC, which indicated that the patients with low miR-891a-5p expression levels had improved overall survival
Table I. Association between miR-891a-5p and the clinical characteristics in patients with non-small cell lung cancer.

\begin{tabular}{|c|c|c|c|c|}
\hline \multirow[b]{2}{*}{ Characteristics } & \multirow[b]{2}{*}{$\begin{array}{l}\text { Total number } \\
\quad(\mathrm{n}=120)\end{array}$} & \multicolumn{2}{|c|}{$\begin{array}{c}\text { miR-891a-5p } \\
\text { expression level }\end{array}$} & \multirow[b]{2}{*}{ P-value } \\
\hline & & $\begin{array}{c}\text { Low } \\
(n=58)\end{array}$ & $\begin{array}{l}\text { High } \\
(n=62)\end{array}$ & \\
\hline Age, years, n & & & & 0.765 \\
\hline$\leq 60$ & 48 & 24 & 24 & \\
\hline$>60$ & 72 & 34 & 38 & \\
\hline Sex, $n$ & & & & 0.916 \\
\hline Female & 47 & 23 & 24 & \\
\hline Male & 73 & 35 & 38 & \\
\hline Smoking status, $\mathrm{n}$ & & & & 0.389 \\
\hline Non-smoker & 49 & 26 & 23 & \\
\hline Smoker & 71 & 32 & 39 & \\
\hline Tumor size, $\mathrm{cm}, \mathrm{n}$ & & & & 0.140 \\
\hline$\leq 3$ & 62 & 34 & 28 & \\
\hline$>3$ & 58 & 24 & 34 & \\
\hline Differentiation, $\mathrm{n}$ & & & & 0.041 \\
\hline Well/moderate & 65 & 37 & 28 & \\
\hline Poor & 55 & 21 & 34 & \\
\hline $\begin{array}{l}\text { Lymph node } \\
\text { metastasis, } \mathrm{n}\end{array}$ & & & & 0.029 \\
\hline Negative & 58 & 34 & 24 & \\
\hline Positive & 62 & 24 & 38 & \\
\hline TNM stage, $\mathrm{n}$ & & & & 0.019 \\
\hline $\mathrm{I} / \mathrm{II}$ & 55 & 33 & 22 & \\
\hline III/IV & 65 & 25 & 40 & \\
\hline
\end{tabular}

miR, microRNA. 


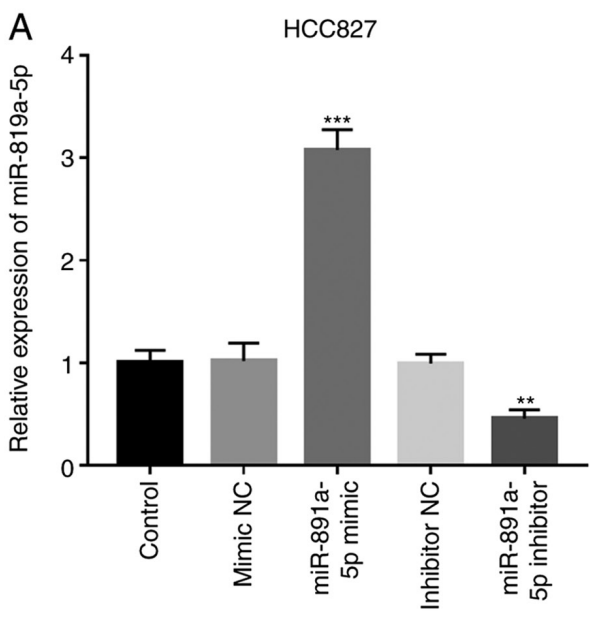

C HCC827

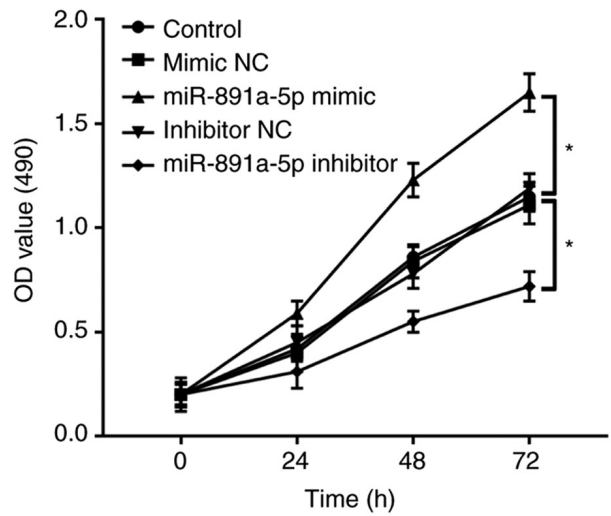

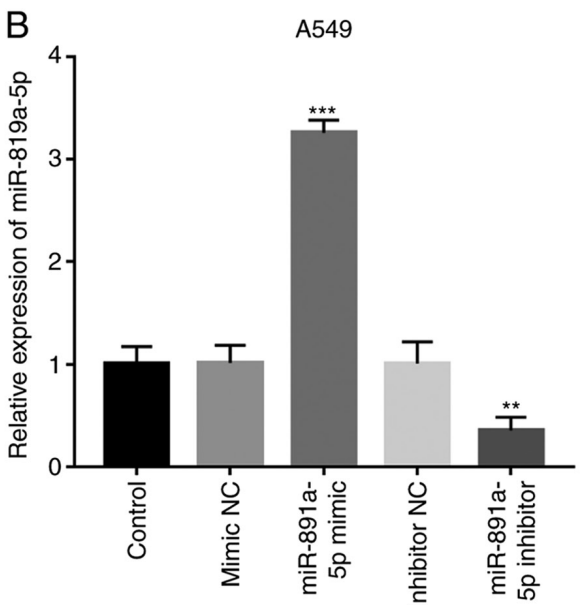

$\mathrm{D}$

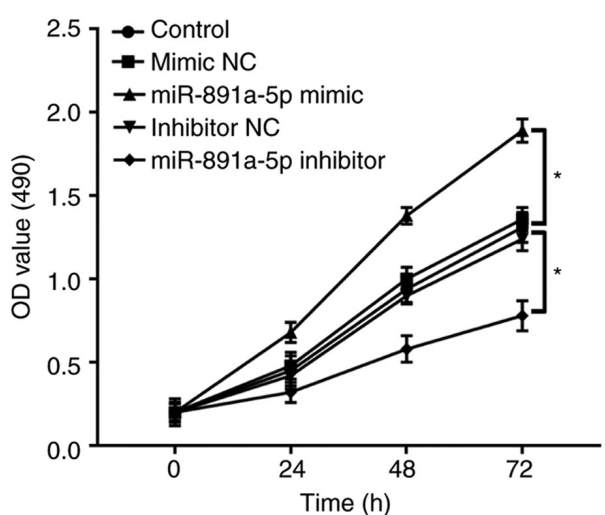

Figure 3. Cell proliferation of the HCC827 and A549 cells following transfection of miR-891a-5p mimics and inhibitor. In (A) HCC827 and (B) A549 cells, the expression level of miR-891a-5p was significantly increased following transfection with miR-891a-5p mimics, but was inhibited following transfection with the miR-891a-5p inhibitor. NSCLC cell proliferation in the (C) HCC827 and (D) A549 cell lines was increased following the transfection of miR-891a-5p mimics, while it was inhibited following the knockdown of miR-891a-5p expression level. ${ }^{*} \mathrm{P}<0.05,{ }^{* *} \mathrm{P}<0.01,{ }^{* * *} \mathrm{P}<0.001$ vs. control. miR, microR $\mathrm{NA}$; NSCLC, non-small cell lung cancer; NC, negative control; OD, optical density.

Table II. Multivariate Cox regression analysis in patients with non-small cell lung cancer.

\begin{tabular}{|c|c|c|c|}
\hline \multirow[b]{2}{*}{ Characteristics } & \multicolumn{3}{|c|}{ Multivariate analysis } \\
\hline & HR & $95 \% \mathrm{CI}$ & P-value \\
\hline miR-819a-5p (high vs. low) & 1.808 & $1.129-2.895$ & 0.014 \\
\hline Age, years (>60 vs. $\leq 60)$ & 1.215 & $0.731-2.020$ & 0.452 \\
\hline Sex (male vs. female) & 1.080 & $0.684-1.706$ & 0.741 \\
\hline Smoking status (yes vs. no) & 1.278 & $0.825-1.981$ & 0.272 \\
\hline Tumor size, cm (>3 vs. $\leq 3$ ) & 1.094 & $0.691-1.731$ & 0.701 \\
\hline Differentiation (yes vs. no) & 1.473 & $0.918-2.362$ & 0.108 \\
\hline $\begin{array}{l}\text { Lymph node metastasis } \\
\text { (yes vs. no) }\end{array}$ & 1.253 & $0.789-1.989$ & 0.339 \\
\hline TNM stage (III/IV vs. I/II) & 1.625 & $1.026-2.573$ & 0.039 \\
\hline
\end{tabular}

HR, hazard ratio.

compared with those with high miR-891a-5p expression levels ( $\log$-rank; $\mathrm{P}=0.015)$. Furthermore, Cox analysis indicated that
miR-891a-5p [hazard ratio (HR), 1.808; 95\% CI, 1.129-2.895; $\mathrm{P}=0.014]$ and TNM stage (HR, 1.625; 95\% CI, 1.026-2.573; $\mathrm{P}=0.039)$ were two independent prognostic factors for the survival of patients with NSCLC (Table II).

Regulatory effects of miR-891a-5p on NSCLC cell proliferation. To further investigate the function of miR-891a-5p in NSCLC tumor progression, in vitro experiments were performed using the HCC827 and A549 cell lines. Following transfection with miR-891a-5p mimics and inhibitors, the mRNA expression level of miR-891a-5p was successfully upregulated and downregulated, respectively, in both the HCC 827 and A549 cell lines (all P<0.001) (Fig. 3A and B). Following which, using a CCK-8 assay, cell proliferation was significantly increased in cells transfected with miR-891a-5p mimics, but was downregulation in cells transfected with the miR-891a-5p inhibitor compared with that in the control (both $\mathrm{P}<0.05$ ) (Fig. 3C and D).

Regulatory effects of miR-891a-5p on NSCLC cell migration and invasion. The effects of miR-891a-5p on cell migration and invasion in the HCC827 and A549 cells were also investigated using a Transwell system. Upregulation of miR-891a-5p 
A
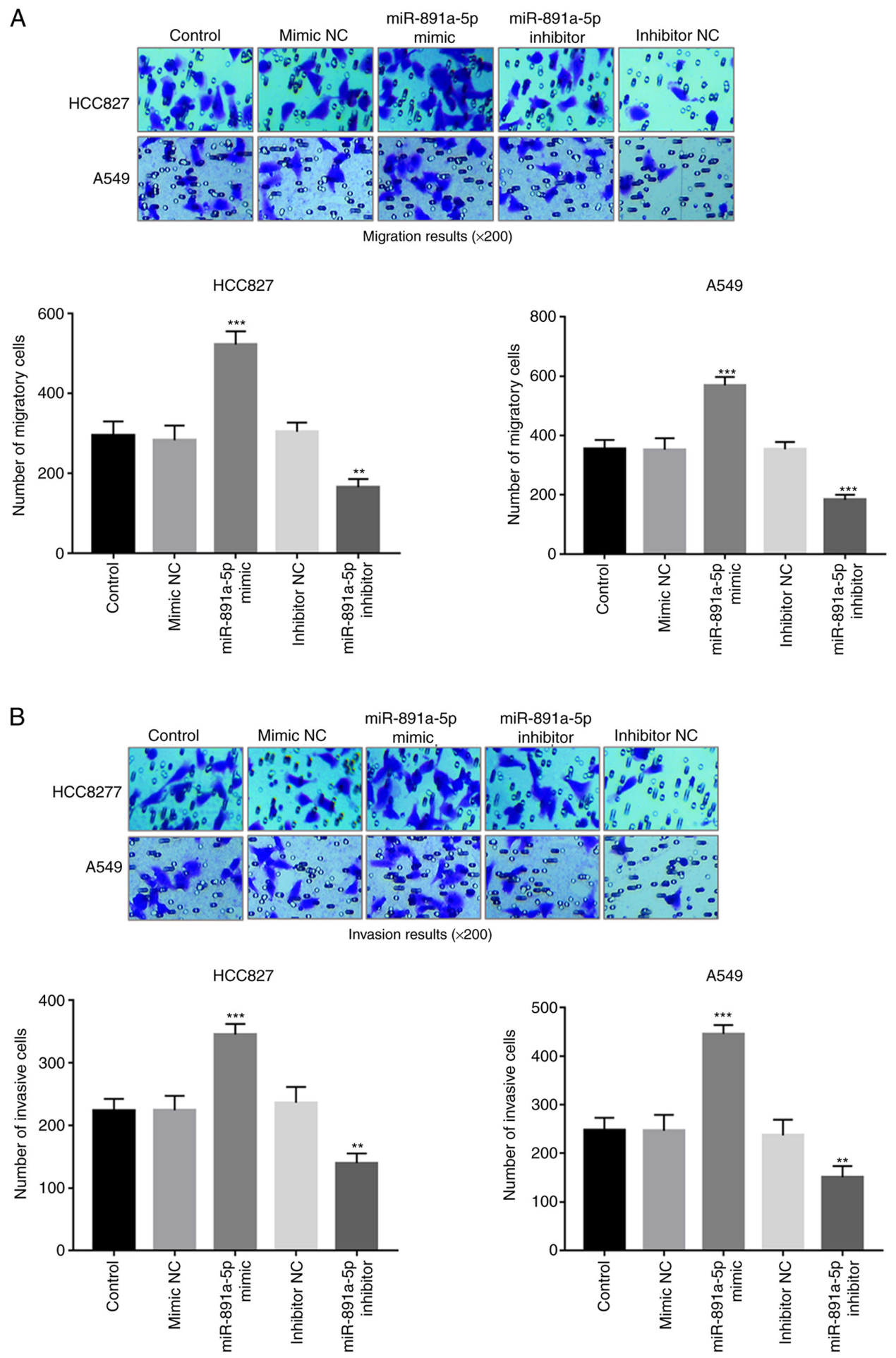

Figure 4. miR-891a-5p promoted the migration and invasion of the HCC827 and A549 cell lines. Overexpression of miR891a-5p enhanced the (A) migration and (B) invasive abilities of the HCC827 and A549 in NSCLC cell lines, but was inhibited by the knockdown of miR-891a-5p. ${ }^{* * *} \mathrm{P}<0.01,{ }^{* * * * *} \mathrm{P}<0.001 \mathrm{vs}$. control. miR, microRNA; NSCLC, non-small cell lung cancer; NC, negative control.

resulted in enhanced cell migration, whereas downregulation of miR-891a-5p resulted in decreased cell migration, in both cell lines (all $\mathrm{P}<0.01$ ) (Fig. 4A). Similarly, the overexpression of miR-891a-5p also promoted the invasive ability of the NSCLC cells, but knockdown of miR-891a-5p inhibited the invasive ability (all $\mathrm{P}<0.01$ ) (Fig. 4B).

HOXA5 is a direct target of miR-891a-5p in the NSCLC cells. According to the results of the TargetScan online analysis,
HOXA5 was predicted to be a target of miR-891a-5p, with a complementary sequence at its 3 '-UTR (Fig. 5A). The binding site for miR-891a-5p was found in the HOXA5 gene, and luciferase reporter assays were used to detect the interaction between miR-891a-5p and HOXA5. A dual-luciferase reporter assay result indicated that the luciferase activity in the HOXA5-WT group was decreased by the upregulated expression level of miR-891a-5p, but was increased by the decreased expression of miR-891a-5p in both the HCC827 and 


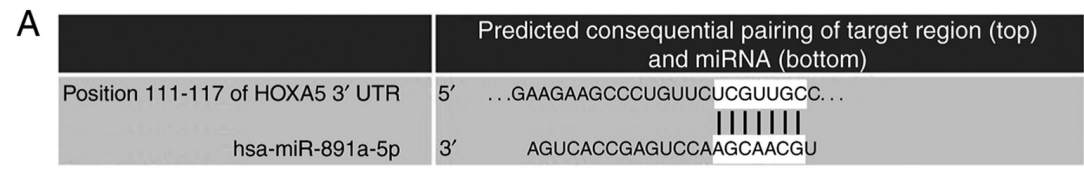

B

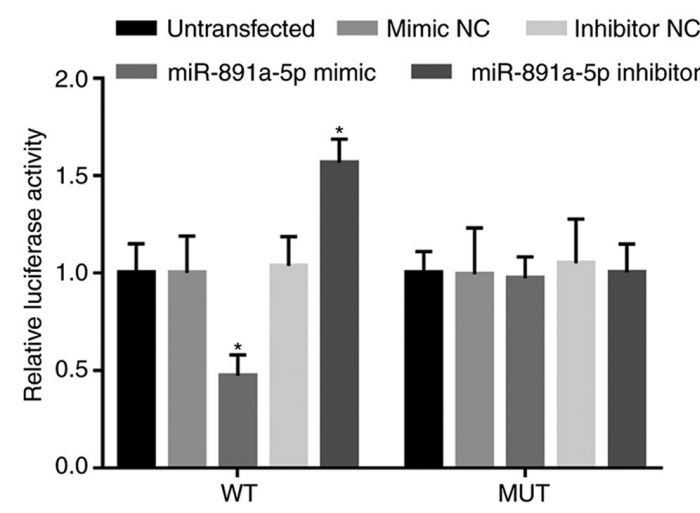

D

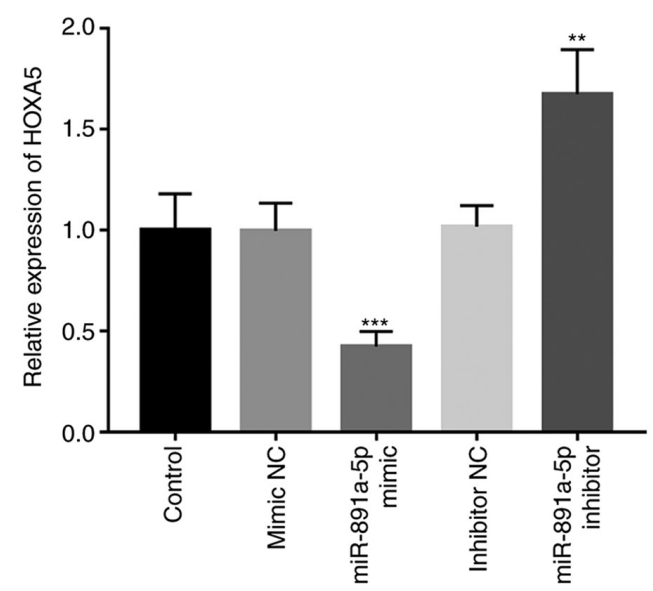

$\mathrm{F}$

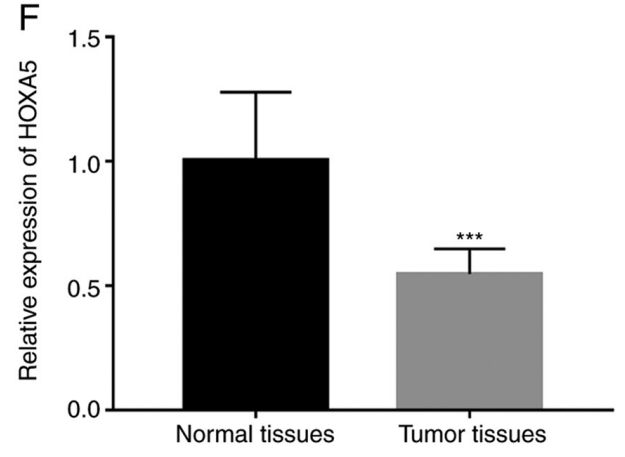

C

A549

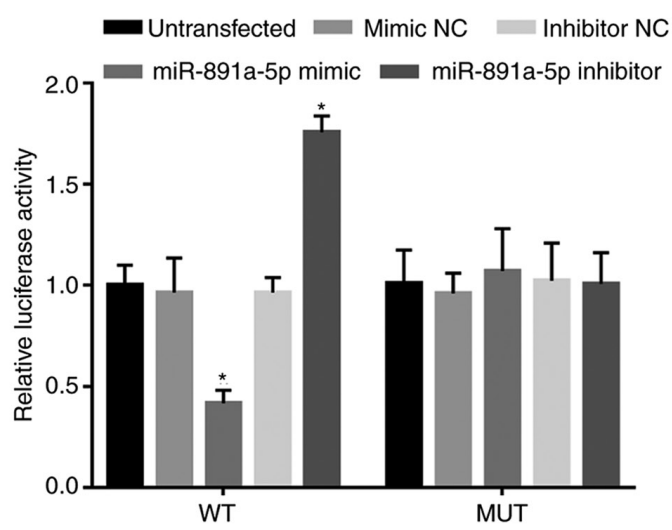

$\mathrm{E}$
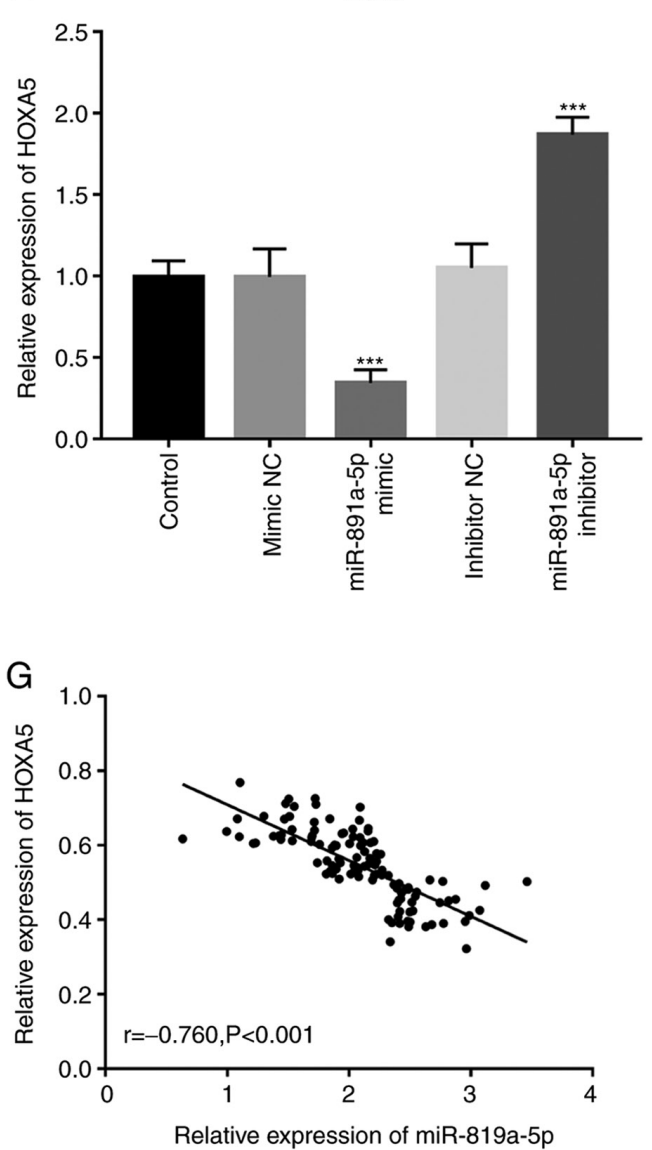

Figure 5. HOXA5 is a direct target of miR-891a-5p in NSCLC cells. (A) The predicted target sequence between HOXA5 and miR-891a-5p. In the (B) HCC827 and (C) A549 NSCLC cell lines, the luciferase activity of the HOXA5-WT was decreased following the overexpression of miR-891a-5p, but was increased by the knockdown of miR-891a-5p expression. "P<0.05 vs. untransfected. The expression level of HOXA5 level in the (D) HCC827 and (E) A549 cells revealed that the miR-891a-5p mimics inhibited the expression level of HOXA5, while knockdown of miR-891a-5p increased the expression level. ${ }^{* *} \mathrm{P}<0.01$, ${ }^{* * *} \mathrm{P}<0.001$ vs. control. (F) The expression level of HOX5 in adjacent normal tissue was significantly increased compared with that in the NSCLC tissue. ${ }^{* * * *} \mathrm{P}<0.001$ vs. normal tissues. (G) The expression level of HOXA5 was negatively correlated with the expression level of miR-891a-5p. miR, microRNA; NSCLC, non-small cell lung cancer; NC, negative control; WT, wild-type; MUT, mutant; UTR, untranslated region.

A549 cells (all $\mathrm{P}<0.05$ ) (Fig. 5B and C). However, there was no significant change in luciferase activity in the MUT group (all $\mathrm{P}>0.05$ ). Detection of HOXA5 mRNA expression levels in the HCC827 and A549 cells revealed that miR-891a-5p mimics inhibited HOXA5 expression levels, whereas silencing of miR-891a-5p enhanced the expression of HOXA5 
(all $\mathrm{P}<0.01$ ) (Fig. 5D and E). In addition, the expression level of HOXA5 in the tumor tissues was significantly lower compared with that in the adjacent normal lung tissues in patients with NSCLC $(\mathrm{P}<0.001$; Fig. 5F), which was found to be negatively correlated with the expression level of miR-891a-5p (r, -0.760; $\mathrm{P}<0.001$; Fig. 5G). The aforementioned results indicated that HOXA5 may be a direct target of miR-891a-5p in NSCLC.

\section{Discussion}

NSCLC is the most common type of lung cancer, worldwide and has numerous pathological features (17). Its estimated 5 -year survival rate is only $15.9 \%$, a figure that has only slightly improved over the past few decades (18). The occurrence and development of NSCLC can result in changes to a wide range of pathological processes, including complex changes in the mRNA expression level of various oncogenes and tumor suppressor genes, such as miR-182-5p, miRNA-148a, miRNA-200a-3p (19-21), that play a key role in cell proliferation and apoptosis (5). Therefore, a deeper understanding of the genes and related mechanisms involved in the progression of NSCLC is urgently required to improve the current treatments and the prognosis of this disease.

miRNA has been shown to be involved in the pathogenesis of several types of cancer, and some miRNA families show functions similar to oncogenes or tumor-suppressor genes (22). For example, it was demonstrated that the expression level of miR-519a was decreased in gastric cancer cell lines compared with that in normal gastric cells, and the proliferation, migration and invasion of tumor cells could be inhibited by the overexpression of miR-519a (23). In osteosarcoma tissues, the expression levels of miR-99b were downregulated and this was associated with the clinical stage and distant metastasis of the patients with osteosarcoma, suggesting it could be used as a biomarker for this type of malignancy (24). Liang et al (25) found that the expression level of miR-146a-5p was increased in triple-negative breast cancer. The aforementioned study showed that patients with high levels of miR-146a-5p in tumor tissues had poor survival, therefore, increased expression of miR-146a-5p in triple-negative breast cancer cells was associated with poor prognosis (25). All the aforementioned studies suggested that functional miRNAs may serve as a new direction into the research for cancer targeted therapy. The role of some miRNA has been investigated in NSCLC, and their clinical significance and functional role have also been determined in previous studies. For example, miR-940 inhibited the proliferation of cancer cells by targeting FAM $83 \mathrm{~F}$ and further inhibited the progression of NSCLC (26). Feng et al (27) found that miR-16-1-3p was significantly downregulated in NSCLC cells compared with that in normal lung cells. In addition, decreased cell proliferation, inhibited cell migration and invasion following transfection with miR-16-1-3p mimics, compared with that in the negative control group.

As a functional miRNA, miR-891a-5p has become a hot topic, particularly when investigating tumors, cell proliferation and cell differentiation (28). Zhang et al found that low expression levels of miR-891a-5p in breast cancer tissue were significantly associated with low distant metastasis-free survival in patients with breast cancer, suggesting that the expression level of miR-891a-5p could be a potential prognostic marker for metastatic human breast cancer (29). However, the biological function and clinical significance of miR-891a-5p have rarely been reported in NSCLC. Therefore, the role of miR-891a-5p in the progression of NSCLC is warranted. In the present study, RT-qPCR was used to determine the mRNA expression levels of miR-891a-5p in NSCLC tissue and serum samples and it was found that the mRNA expression of miR-891a-5p was significantly increased in NSCLC tissue and serum samples compared with that in the corresponding normal controls. Due to the increase in miR-891a-5p mRNA expression level in NSCLC, the clinical significance of miR-891a-5p in the diagnosis and prognosis of NSCLC was also investigated (30). Therefore, ROC analysis was performed based on the serum expression level of miR-891a-5p. The results indicated that the AUC, from the ROC curve, was 0.904, and that an AUC above 0.9 indicated high diagnostic accuracy (31). Thus, miR-891a-5p may have high sensitivity and specificity and could be a potential diagnostic biomarker. According to the Kaplan-Meier survival curves and Cox regression analysis, it was also found that patients with NSCLC and high expression levels of miR-891a-5p had poor overall survival rates and miR-891a-5p was an independent prognostic factor for NSCLC. Furthermore, the increased miR891a-5p expression level was found to be associated with differentiation, lymph node metastasis and TNM stage in patients with NSCLC, indicating that the increased expression level of miR-891a-5p might be associated with the development of NSCLC. However, no significant relationship was found between miR-891a-5p and tumor size, although the regulatory effects of miR-891a-5p on tumor progression were observed in NSCLC cells. In addition, cell proliferation migration and invasion of the tumor cells are not manifested solely by the size of the tumor, but are also associated with the differentiation and TNM stage of the tumor (32). Taken together, it is hypothesized that miR-891a-5p may serve as a candidate diagnostic and prognostic biomarker in patients with NSCLC.

To understand the biological function of miR-891a-5p in the progression of NSCLC, further cellular experiments were performed. The results indicated that the downregulation of miR-891a-5p resulted in suppressed tumor cell migration, proliferation and invasion, but the upregulation of miR-891a-5p led to enhanced tumor cell biological behaviors. The aforementioned results confirmed that miR-891a-5p may play a role in promoting tumorigenesis in NSCLC progression. In addition, the mechanisms of miR-891a-5p in NSCLC was further analyzed. The results of the dual-luciferase reporter assay revealed that miR-891a-5p could directly bind to HOXA5 in NSCLC cell lines. In addition, the results of the cell experiments showed that changing the expression level of cellular miR-891a-5p could affect the expression level of HOXA5 in the cells. Altered HOXA5 methylation levels were found to affect disease development, for example in type II diabetes (33) and colorectal cancer (34). In addition, it was previously shown that HOXA5 was regulated by miRNAs to play a role in numerous types of cancer and affect the biological activity of cancer cells. For example, miR-196a was found to act as a tumor suppressor in gastric cancer by inhibiting cell viability, colony formation, proliferation, cell cycle progression and promoting cell apoptosis by targeting HOXA5 (35). In colon cancer, HOXA5 decreased expression could induce 
the phenotypic loss of tumor stem cells, thereby preventing tumor progression and metastasis (36). HOXA5 expression was altered in patients with different diseases, such as lung cancer, primary pulmonary hypertension and chronic obstructive pulmonary disease (COPD) (37). A previous study by Chang et al (38) showed that overexpression of HOXA5 could reduce the invasive ability of several lung cancer cell lines via a p53-independent pathway. Therefore, it is hypothesized that miR-891a-5p may be involved in NSCLC tumorigenesis by targeting HOXA5.

The present study, has several limitations, such as the limited sample size, the lack of paracancerous tissue results and the absence of animal experiments. In addition, the effects of other factors, such as methylation, on the expression level of HOXA5 were not investigated, which may interfere with the regulation of HOXA5 by miR-891a-5p. Therefore, in future studies, the results from the present study should be confirmed using cancer tissues, paracancerous tissues and adjacent normal tissues from a larger study cohort. The functional role and mechanisms of miR-891a-5p in the tumorigenesis of NSCLC should also be further investigated using animal cancer models, and the specific regulatory mechanism of the HOXA5/miRNA 891a-5p axis.

In conclusion, the results from the present study indicated that miR-891a-5p expression was increased in NSCLC tissues and serum samples, and may be used as a diagnostic and prognostic biomarker. The overexpression of miR-891a-5p was shown to promote NSCLC cell proliferation, migration and invasion, which indicated that miR-891a-5p could be an oncogene in NSCLC progression, and has the potential to be used to improve targeted therapy for NSCLC. HOXA5 was also identified as a target gene of miR-891a-5p, which may mediate the biological function of miR-891a-5p in NSCLC progression.

\section{Acknowledgements}

Not applicable.

\section{Funding}

No funding was received.

\section{Availability of data and materials}

All data generated or analyzed during this study are included in this published article.

\section{Authors' contributions}

NW and JZ designed the study, performed clinical studies, analyzed data and wrote the manuscript. NW performed the cell experiments. NW and JZ confirm the authenticity of all raw data. All authors have read and approved the final manuscript.

\section{Ethics approval and consent to participate}

Written informed consent was provided by each patient and the experimental procedures were all in accordance with the guidelines of the Ethics Committee of Yueqing People's Hospital (Yueqing, China; approval no. 0112983).

\section{Patient consent for publication}

Not applicable.

\section{Competing interests}

The authors declare that they have no competing interests.

\section{References}

1. Nasim F, Sabath BF and Eapen GA: Lung Cancer. Med Clin North Am 103: 463-473, 2019.

2. Võsa U, Vooder T, Kolde R, Vilo J, Metspalu A and Annilo T: Meta-analysis of microRNA expression in lung cancer. Int $\mathrm{J}$ Cancer 132: 2884-2893, 2013.

3. Chen W, Zheng R, Baade PD, Zhang S, Zeng H, Bray F, Jemal A, Yu XQ and He J: Cancer statistics in China, 2015. CA Cancer J Clin 66: 115-132, 2016.

4. Torre LA, Bray F, Siegel RL, Ferlay J, Lortet-Tieulent J and Jemal A: Global cancer statistics, 2012. CA Cancer J Clin 65: 87-108, 2015.

5. Liu J, Bian T, Feng J, Qian L, Zhang J, Jiang D, Zhang Q, Li X, Liu Y and Shi J: miR-335 inhibited cell proliferation of lung cancer cells by target Tra2 $\beta$. Cancer Sci 109: 289-296, 2018.

6. Wu YL, Sun Y, Zhou CC, Zhang L, Yu SY, Ma SL, Han LL, Zhang XQ and Orlando M: Survival without common toxicity criteria grade $3 / 4$ toxicity following second-line treatment with pemetrexed for nonsquamous non-small cell lung cancer in Chinese patients. Chin Med J (Engl) 126: 4624-4628, 2013.

7. Woodard GA, Jones KD and Jablons DM: Lung Cancer Staging and Prognosis. Cancer Treat Res 170: 47-75, 2016.

8. Turchinovich A, Weiz L and Burwinkel B: Extracellular miRNAs: The mystery of their origin and function. Trends Biochem Sci 37: 460-465, 2012.

9. Barbato S, Solaini G and Fabbri M: MicroRNAs in oncogenesis and tumor suppression. Int Rev Cell Mol Biol 333: 229-268, 2017.

10. Zhang X, Xu X, Ge G, Zang X, Shao M, Zou S, Zhang Y, Mao Z, Zhang J, Mao F, et al: miR 498 inhibits the growth and metastasis of liver cancer by targeting ZEB2. Oncol Rep 41: 1638-1648, 2019.

11. Yuan J, Su Z, Gu W, Shen X, Zhao Q, Shi L, Jin C, Wang X, Cong $\mathrm{H}$ and Ju S: MiR-19b and miR-20a suppress apoptosis, promote proliferation and induce tumorigenicity of multiple myeloma cells by targeting PTEN. Cancer Biomark 24: 279-289, 2019.

12. Ganju A, Khan S, Hafeez BB, Behrman SW, Yallapu MM, Chauhan SC and Jaggi M: miRNA nanotherapeutics for cancer. Drug Discov Today 22: 424-432, 2017.

13. Lin K, Xu T, He BS, Pan YQ, Sun HL, Peng HX, Hu XX and Wang SK: MicroRNA expression profiles predict progression and clinical outcome in lung adenocarcinoma. OncoTargets Ther 9: 5679-5692, 2016.

14. Lee HY, Han SS, Rhee H, Park JH, Lee JS, Oh YM, Choi SS, Shin SH and Kim WJ: Differential expression of microRNAs and their target genes in non-small-cell lung cancer. Mol Med Rep 11: 2034-2040, 2015.

15. Livak KJ and Schmittgen TD: Analysis of relative gene expression data using real-time quantitative PCR and the 2(-Delta Delta C(T)) method. Methods 25: 402-408, 2001.

16. Lin Z, Zhao J, Wang X, Zhu X and Gong L: Overexpression of microRNA-497 suppresses cell proliferation and induces apoptosis through targeting paired box 2 in human ovarian cancer. Oncol Rep 36: 2101-2107, 2016.

17. Chen Z, Fillmore CM, Hammerman PS, Kim CF and Wong KK: Non-small-cell lung cancers: A heterogeneous set of diseases. Nat Rev Cancer 14: 535-546, 2014.

18. Akhurst T: Staging of non-small-cell lung cancer. PET Clin 13: 1-10, 2018.

19. Gao L, Yan SB, Yang J, Kong JL, Shi K, Ma FC, Huang LZ, Luo J, Yin SY, He RQ, et al: MiR-182-5p and its target HOXA9 in non-small cell lung cancer: A clinical and in-silico exploration with the combination of RT-qPCR, miRNA-seq and miRNA-chip. BMC Med Genomics 13: 3, 2020. 
20. Chen Y, Min L, Ren C, Xu X, Yang J, Sun X, Wang T, Wang F, Sun $C$ and Zhang X: miRNA-148a serves as a prognostic factor and suppresses migration and invasion through Wnt1 in non-small cell lung cancer. PLoS One 12: e0171751, 2017.

21. Tan T, Xu XH, Lu XH and Wang XW: MiRNA-200a-3p suppresses the proliferation, migration and invasion of non-small cell lung cancer through targeting IRS2. Eur Rev Med Pharmacol Sci 24: 712-720, 2020.

22. Zhang L, Deng T, Li X, Liu H, Zhou H, Ma J, Wu M, Zhou M, Shen S, Li X, et al: microRNA-141 is involved in a nasopharyngeal carcinoma-related genes network. Carcinogenesis 31: $559-566,2010$

23. Cai H, Lin H, Cao W, Sun J, Huang Y and Fang Y: The downregulation of miR-519a predicts poor prognosis and contributes to tumor progression in gastric cancer. Int J Clin Exp Pathol 12: 2496-2505, 2019.

24. Shi X and Guan X: MicroRNA-99b predicts clinical outcome of osteosarcoma and suppresses tumor cell proliferation, migration and invasion. Diagn Pathol 14: 117, 2019.

25. Liang H, Huang W, Wang Y, Ding L and Zeng L: Overexpression of MiR-146a-5p Upregulates lncRNA HOTAIR in triple-negative breast cancer cells and predicts poor prognosis. Technol Cancer Res Treat 18: 1533033819882949,2019.

26. Gu GM, Zhan YY, Abuduwaili K, Wang XL, Li XQ, Zhu HG and Liu CL: MiR-940 inhibits the progression of NSCLC by targeting FAM83F. Eur Rev Med Pharmacol Sci 22: 5964-5971, 2018.

27. Feng QQ, Dong ZQ, Zhou Y, Zhang H and Long C: miR-16-1-3p targets TWIST1 to inhibit cell proliferation and invasion in NSCLC. Bratisl Lek Listy 119: 60-65, 2018.

28. Yao S, Hu M, Hao T, Li W, Xue X, Xue M, Zhu X, Zhou F, Qin D, Yan Q, et al: MiRNA-891a-5p mediates HIV-1 Tat and KSHV Orf-K1 synergistic induction of angiogenesis by activating NF- $\kappa$ B signaling. Nucleic Acids Res 47: 2700, 2019.

29. Zhang Z, Xu L, He L, Wang J, Shi X, Li Z, Shi S, Hou K, Teng Y and Qu X: MiR-891a-5p as a prognostic marker and therapeutic target for hormone receptor-positive breast cancer. J Cancer 11: 3771-3782, 2020.
30. Lu X and Lu J: The significance of detection of serum miR-423-5p and miR-484 for diagnosis of colorectal cancer. Clin Lab 61: $187-190,2015$

31. Kamarudin AN, Cox T and Kolamunnage-Dona R: Time-dependent ROC curve analysis in medical research: Current methods and applications. BMC Med Res Methodol 17: 53, 2017.

32. Enane FO, Saunthararajah Y and Korc M: Differentiation therapy and the mechanisms that terminate cancer cell proliferation without harming normal cells. Cell Death Dis 9: 912, 2018

33. Cao W, Xu Y, Luo D, Saeed M and Sun C: Hoxa5 promotes adipose differentiation via increasing DNA methylation level and inhibiting PKA/HSL signal pathway in Mice. Cell Physiol Biochem 45: 1023-1033, 2018.

34. Li D, Bai Y, Feng Z, Li W, Yang C, Guo Y, Lin C, Zhang Y, He Q, $\mathrm{Hu} \mathrm{G}$, et al: Study of promoter methylation patterns of HOXA2, HOXA5, and HOXA6 and its clinicopathological characteristics in colorectal cancer. Front Oncol 9: 394, 2019.

35. Wu Y, Zhou T, Tang Q and Xiao J: HOXA5 inhibits tumor growth of gastric cancer under the regulation of microRNA-196a. Gene 681: 62-68, 2019.

36. Ordóñez-Morán P, Dafflon C, Imajo M, Nishida E and Huelsken J: HOXA5 counteracts stem cell traits by inhibiting Wnt signaling in colorectal cancer. Cancer Cell 28: 815-829, 2015.

37. Liu XH, Lu KH, Wang KM, Sun M, Zhang EB, Yang JS, Yin DD, Liu ZL, Zhou J, Liu ZJ, et al: MicroRNA-196a promotes non-small cell lung cancer cell proliferation and invasion through targeting HOXA5. BMC Cancer 12: 348, 2012.

38. Chang CJ, Chen YL, Hsieh CH, Liu YJ, Yu SL, Chen JJ and Wang CC: HOXA5 and p53 cooperate to suppress lung cancer cell invasion and serve as good prognostic factors in non-small cell lung cancer. J Cancer 8: 1071-1081, 2017.

This work is licensed under a Creative Commons Attribution-NonCommercial-NoDerivatives 4.0 International (CC BY-NC-ND 4.0) License. 